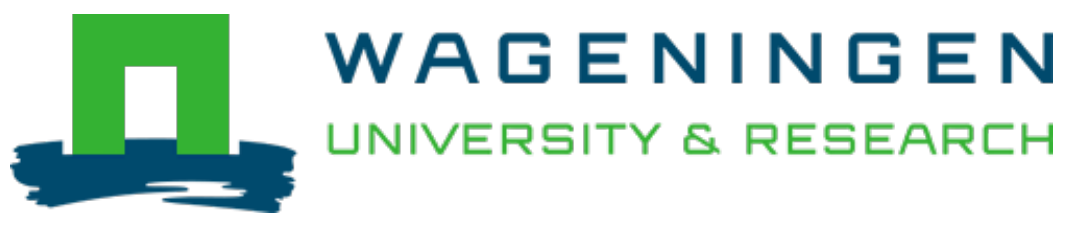

\title{
Microstructural changes during alkali- and heat induced gelation of konjac glucomannan
}

\author{
Food Hydrocolloids \\ Zhang, Tao; Vries, Renko; Xu, Xiaoqi; Xue, Yong; Xue, Changhu \\ https://doi.org/10.1016/j.foodhyd.2020.106552
}

This article is made publicly available in the institutional repository of Wageningen University and Research, under the terms of article $25 \mathrm{fa}$ of the Dutch Copyright Act, also known as the Amendment Taverne. This has been done with explicit consent by the author.

Article 25 fa states that the author of a short scientific work funded either wholly or partially by Dutch public funds is entitled to make that work publicly available for no consideration following a reasonable period of time after the work was first published, provided that clear reference is made to the source of the first publication of the work.

This publication is distributed under The Association of Universities in the Netherlands (VSNU) 'Article $25 \mathrm{fa}$ implementation' project. In this project research outputs of researchers employed by Dutch Universities that comply with the legal requirements of Article $25 \mathrm{fa}$ of the Dutch Copyright Act are distributed online and free of cost or other barriers in institutional repositories. Research outputs are distributed six months after their first online publication in the original published version and with proper attribution to the source of the original publication.

You are permitted to download and use the publication for personal purposes. All rights remain with the author(s) and / or copyright owner(s) of this work. Any use of the publication or parts of it other than authorised under article $25 \mathrm{fa}$ of the Dutch Copyright act is prohibited. Wageningen University \& Research and the author(s) of this publication shall not be held responsible or liable for any damages resulting from your (re)use of this publication.

For questions regarding the public availability of this article please contact openscience.library@,wur.nl 


\title{
Microstructural changes during alkali- and heat induced gelation of konjac glucomannan
}

\author{
Tao Zhang ${ }^{\mathrm{a}, \mathrm{b}}$, Renko de Vries ${ }^{\mathrm{b}, * *}$, Xiaoqi Xu ${ }^{\mathrm{c}, * * *}$, Yong Xue ${ }^{\mathrm{d}, *}$, Changhu Xue ${ }^{\mathrm{d}}$ \\ ${ }^{a}$ College of Food Science and Engineering, Nanjing University of Finance and Economics, Nanjing, 210023, China \\ ${ }^{\mathrm{b}}$ Physical Chemistry and Soft Matter, Wageningen University \& Research, Stippeneng 4, 6708 WE, Wageningen, the Netherlands \\ c College of Food and Light Industry, Nanjing Tech University, Nanjing, 211800, China \\ ${ }^{\mathrm{d}}$ Department of Food Science and Engineering, Ocean University of China, Qingdao, 266003, China
}

\section{A R T I C L E I N F O}

\section{Keywords:}

Konjac glucomannan

Gelation

Framework

Particle size

Atomic force microscopy

\begin{abstract}
A B S T R A C T
We investigate structural changes of konjac glucomannan (KGM) during heat induced gelation of predeacetylated samples. We combine rheology at higher concentrations, with light scattering and AFM studies on low concentration samples. Macroscale rheology demonstrates that gelation requires both heat and alkaliinduced deacetylation. With Dynamic Light Scattering (DLS) we investigated the time evolution of KGM particle hydrodynamic radius $(R h)$ during the deacetylation reaction. Before deacetylation we observe that heating leads to swelling of the KGM particles in solution. From the kinetics of particle aggregation, we deduce that deacetylation itself is the rate-determining step and that aggregation occurs immediately after deacetylation. In Atomic Force Microscopy (AFM), native KGM particles appear as compact globular objects. Consistent with the light scattering results, we observe that heating leads to opening and swelling of these particles, and that deacetylation leads to their interaction to each other into a network. Our DLS and AFM results help understand the microscopic origins of heat- and alkali-induced gelation of KGM.
\end{abstract}

\section{Introduction}

Konjac glucomamnan (KGM), is a high molecular weight, neutral polysaccharide extracted from the tubers of Amorphophallus konjac plants, a member of the family Araceae found in east Asia (Nishinari, Williams, \& Phillips, 1992). The main chain of KGM mainly consists of D-mannose and D-glucose units through $\beta$-1,4-linkage in a mole ratio of 1.6:1 (Maeda, Shimahara, \& Sugiyama, 1980) or 1.4:1 (Dey \& Dixon, 1985). Acetyl groups attached to the saccharide units are scattered randomly along the molecule, at approximately 1 acetyl group per 19 sugar residues at the C-6 position (Katsuraya et al., 2003). At alkaline conditions, deacetylation leads to irreversible association of the KGM molecules (Du, Li, Chen, \& Li, 2012; Gao \& Nishinari, 2004). This process has been studied by many research groups not only from a scientific interest but also in view of applications in the food industry, pharmaceutical, biomedical, cosmetic, coating, painting, and related industries (Huang, Takahashi, Kobayashi, Kawase, \& Nishinari, 2002).

An early study by Maekaji (Maekaji, 1974) concluded that, by losing their acetyl groups at alkaline conditions, KGM molecules associate to each other via hydrogen bonds, leading to gel formation. More recently it has also been emphasized that deacetylated KGM has a hydrophobic character. This was concluded among others from the dependence of KGM hydrogel rheology on the presence of lyotropic series of salts (Case, Knopp, Hamann, \& Schwartz, 1992; Chen., Li, \& Li, 2011; Nishinari \& Zhang, 2004). A similar conclusion was drawn from fluorescence measurements on KGM gelation at different DD (Xin et al., 2017). To obtain an integrated picture that connects observations across multiple timeand length scales, Williams et al. (2000) have studied KGM gelation through a combination of rheology and NMR. Nevertheless, even these authors have to conclude that "a detailed dynamical molecular level picture of the aggregation process is lacking". Additionally, various kinetic models have been proposed for the time-dependent buildup of network elasticity by including the key steps of deacetylation and the subsequent association of the KGM molecules to form a network (Gao \& Nishinari, 2004; Mao \& Chen, 2017).

The conformational changes to the KGM molecules upon gelation

\footnotetext{
* Corresponding author.

$* *$ Corresponding author.

$* * *$ Corresponding author.

E-mail addresses: renko.devries@wur.nl (R. de Vries), xiaoqi_xu@njtech.edu.cn (X. Xu), xueyong@ouc.edu.cn (Y. Xue).
} 
have not yet been elucidated completely. Some authors (Jian, Siu, \& Wu, 2015) find that chain sizes of KGM in solution strongly depend on solution conditions, but that chain conformations are always random coils. It appears (Zhou, Jiang, Perkins, \& Cheng, 2018) that the irreversible formation of large KGM fiber bundles is preceded by a self-assembly step in which random coils assemble to form thin filaments.

While there is now a fair body of work on alkali induced gelation of KGM, an integrated picture that connects observations across multiple time- and length scales, from molecular to macroscale, would be helpful for obtaining a deeper understanding of KGM gelation. With this in mind we here combine macroscale observations from rheology with mesoscale and microscale observations from Dynamic Light Scattering and Atomic Force Microscopy, to obtain such a picture for the alkali-induced gelation of KGM.

\section{Materials and methods}

\subsection{Materials}

Commercial konjac flour (KGM $>90 \mathrm{wt} \%$ ) was purchased from Chengdu Xifu Biological Technology Co., Ltd. (Chengdu, Sichuan Province, China). All the chemicals were of analytical grade, unless otherwise specified.

\subsection{Purification of $K G M$}

Purification and Deacetylation of KGM was done according to the method of Jian et al. (2013) with some modifications. Konjac flour

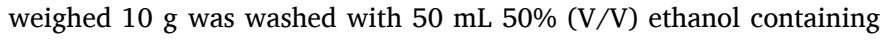
$0.1 \%$ sodium azide for three times so as to remove water-soluble impurity. After drying, the powder was degreased in $50 \mathrm{~mL}$ mixed solvent of absolute diethyl ether/absolute ethanol $(2: 1 ; \mathrm{V} / \mathrm{V})$ at $40{ }^{\circ} \mathrm{C}$ under stirring for $8 \mathrm{~h}$. Next, the degreased sample was dissolved in distilled water at a concentration of $0.6 \%$. Water insoluble impurities were removed by centrifugation (Heraeus Multifuge X1R, Thermo Fisher Scientific, Waltham, USA) at a centrifugal force of $18,000 \times g$ for $20 \mathrm{~min}$. Then, amylase $(0.5 \mathrm{~g} / \mathrm{L})$ was added to remove starch at room temperature until enzymatic hydrolysis was complete. Next, deproteinization was carried out following the Sevag method (Li \& Xie, 2006) for five times, followed by centrifugation at $18,000 \times g$ for $20 \mathrm{~min}$. To the supernatant, the same volume of ethanol $(95 \%, \mathrm{~V} / \mathrm{V})$ was added to precipitate the KGM. The precipitate was washed with absolute ethanol and ether. Finally, the precipitate was freeze dried to obtain pure KGM powder.

\subsection{Preparation of KGM samples with different degree of deacetylation}

Preparation of KGM samples with different degree of deacetylation was done according to the method of (Du et al., 2012) with some modifications. KGM ( $10 \mathrm{~g}$ ) was mixed with $50 \mathrm{ml}$ of $50 \mathrm{vol} \%$ ethanol in a water bath shaker at $150 \mathrm{rpm}$ and $40{ }^{\circ} \mathrm{C}$ for $30 \mathrm{~min}$. A predetermined amount of $\mathrm{Na}_{2} \mathrm{CO}_{3}$ was added immediately into it. The deacetylation reaction was performed at $40{ }^{\circ} \mathrm{C}$ and allowed to proceed for $24 \mathrm{~h}$. The crude product was immersed into twice the volume of $50 \mathrm{vol} \%$ ethanol, and this was repeated several times until the sample $\mathrm{pH}$ became neutral. Afterward, the product was dehydrated gradually with 75 vol\% aqueous ethanol and 95 vol\% aqueous ethanol, and pure ethanol. The final products were obtained by vacuum-drying at $40{ }^{\circ} \mathrm{C}$. By changing the amount of $\mathrm{Na}_{2} \mathrm{CO}_{3}$, a series of deacetylated KGM with different DDs were obtained.

The degrees of deacetylation of KGM corresponding to the different amounts of $\mathrm{Na}_{2} \mathrm{CO}_{3}$ added were determined through the a titration method (Chen, Zong, \& Li, 2006). Briefly, a dried sample of powdered KGM was placed in a $100 \mathrm{ml}$ Erlenmeyer flask with a stopper and $75 \%$ ethanol $(10 \mathrm{ml})$ was added. The mixture was stirred at $40^{\circ} \mathrm{C}$ for $30 \mathrm{~min}$, followed by adding $\mathrm{Na}_{2} \mathrm{CO}_{3}$ with swirling. The temperature was maintained at $40^{\circ} \mathrm{C}$ for $24 \mathrm{~h}$ with stirring. Excess alkali was back titrated with $0.1 \mathrm{M} \mathrm{HCl}$ using phenolphthalein as an indicator. A blank (lacking KGM) was titrated in parallel. The content of acetyl $(\omega, w / w)$ was calculated according to the following equation:

$\omega=\frac{\left(\mathrm{V}_{2}-\mathrm{V}_{1}\right) \times \mathrm{N}_{\mathrm{HCl}} \times \mathrm{M}_{\text {acetyl }}}{\mathrm{m}_{\mathrm{s}}} \times 100 \%$

where $V_{2}$ is the volume of hydrochloric acid consumed for the blank in liters, $V_{1}$ represents the volume of hydrochloric acid consumed for the sample in liters, $N_{\mathrm{HCl}}$ stands for the normality of the hydrochloric acid, $M_{\text {acetyl }}=43 \mathrm{~g} / \mathrm{mol}$ and $m_{s}$ is the mass of the sample in grams. By changing the amount of $\mathrm{Na}_{2} \mathrm{CO}_{3}$ and controlling reaction time, different DDs of KGM were obtained:

$\mathrm{DD}=\frac{\omega_{0}-\omega}{\omega_{0}} \times 100 \%$

where $\omega_{0}$ is the content of acetyl of native KGM (w/w), and $\omega$ is the content of acetyl after the deacetylation reaction.

The content of acetyl of native KGM was established to be $\omega_{0}=$ $1.82 \%$. A series of samples at different DDs was prepared reaction of native KGM with varying amounts of $\mathrm{Na}_{2} \mathrm{CO}_{3}$. The samples were labeled as $\mathrm{Da} 0-\mathrm{Da} 5$. The respective degrees of deacetylation were found are $\mathrm{Da} 0$ : $\mathrm{DD}=0.0 \%$ (native KGM), Da1: $\mathrm{DD}=16.13 \pm 0.38 \%, \mathrm{Da} 2 \mathrm{DD}=34.13$ $\pm 0.21 \%$, Da3: DD $=65.84 \pm 0.34 \%$, Da4: DD $=76.13 \pm 0.46 \%$, and Da5: $\mathrm{DD}=98.29 \pm 0.17 \%$. All data are averages of experiments performed at least in duplicate. Reaction products were also analyzed by SEC-MALLS (SI Figs. S1 and SI Table S1). Deacetylation did not lead to significant degradation of the KGM, with molecular weights Mw of the samples decreasing only somewhat, from $7.8 \cdot 10^{5} \mathrm{~g} / \mathrm{mol}$ for Da0 to $6.3 \cdot 10^{5} \mathrm{~g} / \mathrm{mol}$ for Da5. Using FT-IR spectroscopy, it was confirmed that the Da0-Da5 KGM samples had been deacetylated to varying degrees (SI Fig. S2).

\subsection{Rheology}

The rheological behavior of the KGM samples was characterized using an MCR 301 rheometer (Anton Paar, Austria) with a stainless-steel parallel plate geometry ( $50 \mathrm{~mm}$ diameter), and gap size of $1.0 \mathrm{~mm}$ for all experiments. A thin layer of paraffin was applied on the edge of the sample, in order to prevent sample evaporation. To determine the linear viscoelastic (LVE) region for each sample, initial strain sweep tests were performed from $0.01 \%$ to $100 \%$ at $1 \mathrm{~Hz}$ and at $25{ }^{\circ} \mathrm{C}$. It was found that strain of $0.5 \%$ was well within the linear regime and gave reproducible results.

In a first type of experiment (temperature ramp), $\mathrm{Na}_{2} \mathrm{CO}_{3}$ was added to $1 \%(\mathrm{w} / \mathrm{v})$ native KGM. The sample was immediately applied on the bottom plate of the rheometer. Next, a measurement was started in which the stress was monitored during oscillatory strain $\gamma(0.5 \%)$ at a fixed frequency of $\omega=1 \mathrm{~Hz}$. During the measurement, the temperature $T$ was increased linearly, with a heating rate of $1{ }^{\circ} \mathrm{C} / \mathrm{min}$, in a temperature range from $10{ }^{\circ} \mathrm{C}$ to $90{ }^{\circ} \mathrm{C}$. From the data, we obtained the storage modulus $G^{\prime}(\omega)$ and loss modulus $G^{\prime \prime}(\omega)$ as a function of temperature, as well as the loss factor $\tan \delta$ (Winter \& Chambon, 1986):

$\tan \delta=\frac{\mathrm{G}^{\prime \prime}(\omega)}{\mathrm{G}^{\prime}(\omega)}$

In another type of experiment (fixed temperature), which was performed only for the Da5 sample, rather than using a temperature ramp, after mixing the native $\mathrm{KGM}$ with the $\mathrm{Na}_{2} \mathrm{CO}_{3}$, the sample was rapidly (5 ${ }^{\circ} \mathrm{C} / \mathrm{min}$ ) heated (or cooled) to a fixed temperature and the storage and loss moduli were measured as a function of time, again at fixed strain $(0.5 \%)$ and oscillation frequency $(\omega=1 \mathrm{~Hz})$. This experiment was repeated for a number of temperatures. 

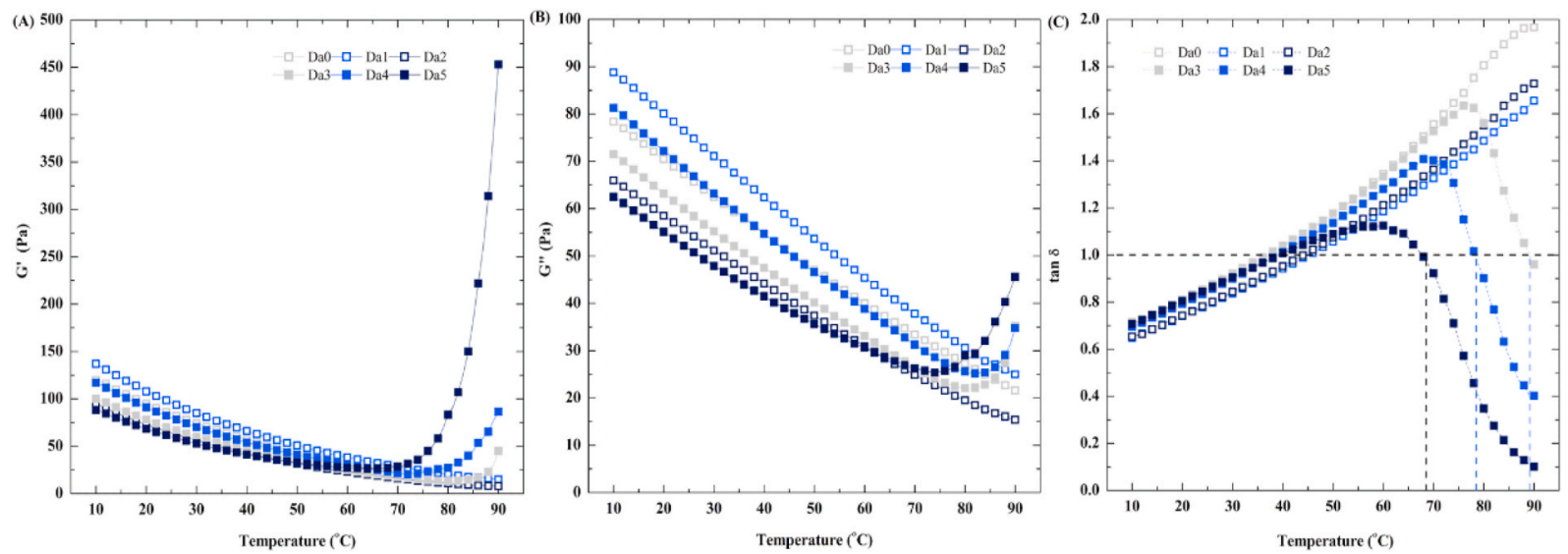

Fig. 1. (A) Storage modulus $G^{\prime}(\omega)$, (B) Loss modulus $G^{\prime \prime}(\omega)$ and (C) Loss factor tan $\delta=G^{\prime \prime} / G^{\prime}$, for KGM (1 wt $\%$ ) samples with different degrees of deacetylation as a function of temperature $T$. Measurements at fixed frequency $\omega=1 \mathrm{~Hz}$ during a heating ramp with constant heating rate of $1{ }^{\circ} \mathrm{C} / \mathrm{min}$, for samples to which different amounts of alkali have been added at the start of the heating ramp, corresponding to different final degrees of deacetylation, as indicated in the figure (Da0-Da5).
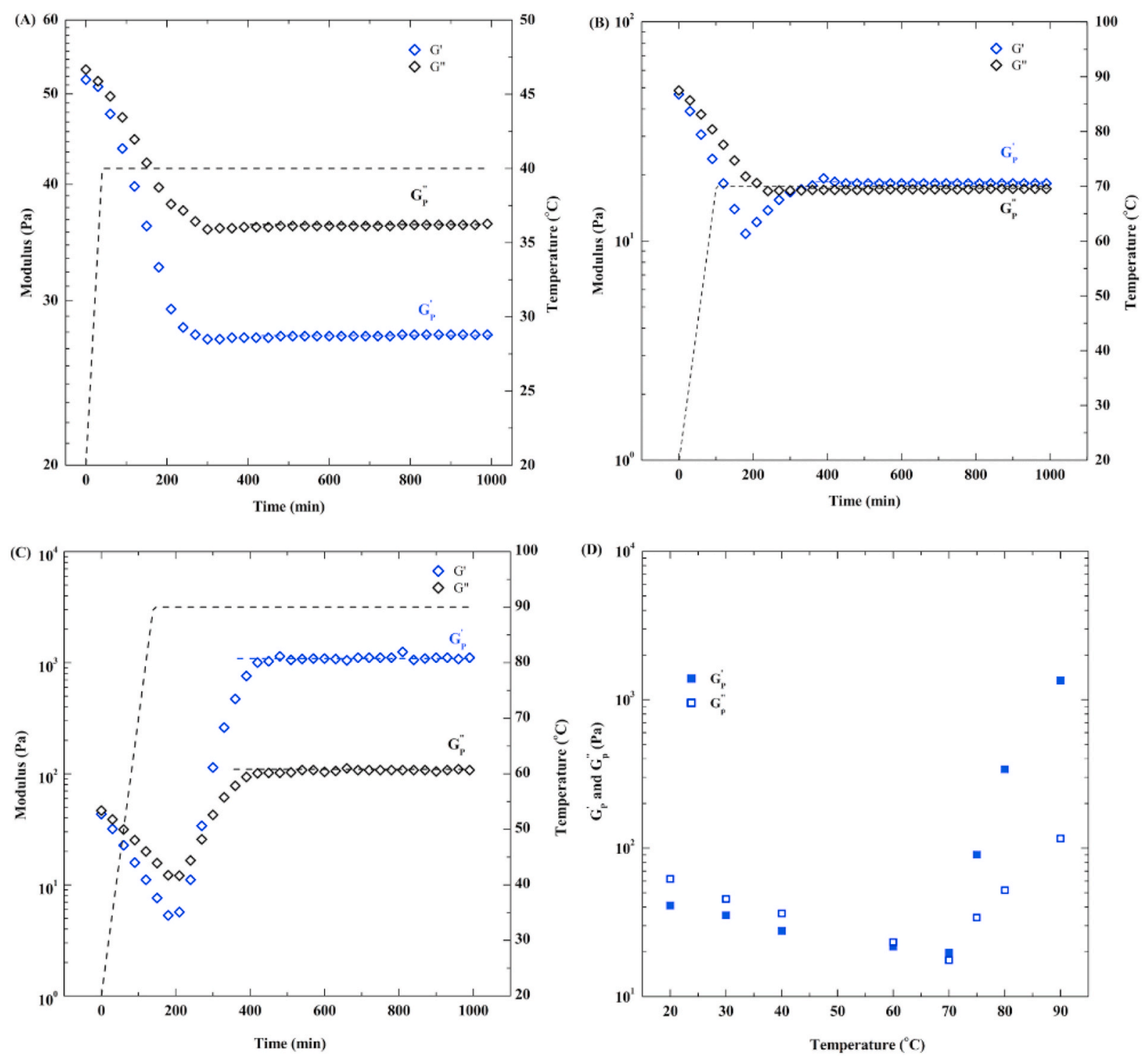

Fig. 2. Gel formation for Da5 ( $1 \mathrm{wt} \%)$ during deacetylation reaction at fixed temperature $T_{f}$ (after rapid heating at $5{ }^{\circ} \mathrm{C} / \mathrm{min}$ ). Storage and loss moduli $G^{\prime}(\omega)$ and $G^{\prime \prime}$ $(\omega) \mathrm{Da} 5(1 \mathrm{wt} \%)$ at $\omega=1 \mathrm{~Hz}$ as a function of reaction time for specific temperature (A) $T_{f}=40^{\circ} \mathrm{C}$, (B) $T_{f}=70^{\circ} \mathrm{C}$, and (C) $T_{f}=90^{\circ} \mathrm{C}$. (D) Final storage and loss moduli $G^{\prime}(\omega)$ and $G^{\prime \prime}(\omega)$ of Da5 $(1 \mathrm{wt} \%)$ at $\omega=1 \mathrm{~Hz}$ as a function of the fixed reaction temperature $T_{f}$. 


\subsection{Dynamical light scattering (DLS)}

Dynamic light scattering (DLS) was performed using a Zetasizer Nano ZS (ZEN3600, Malvern Instruments Limited, UK), with He-Ne laser $(633 \mathrm{~nm}, 4.0 \mathrm{~mW})$ at scattering angle of $173^{\circ}$. For the light scattering experiments, KGM samples were diluted to a very low concentration of $0.01 \%(\mathrm{w} / \mathrm{v})$ such that only KGM aggregation but not KGM gelation is expected after the addition of $\mathrm{Na}_{2} \mathrm{CO}_{3}$. Each measurement is was obtained by averaging data from at least 5 runs.

Measurements were analyzed using DTS Software version 7.02 (Malvern Instruments Limited, UK) to obtain the z-averaged hydrodynamic radius $\left(R_{h}\right)$ of the KGM particles in solution. The z-averaged hydrodynamic radius is the average that follows naturally from the scattering intensity-weighted diffusion constant. It is a very stable reporter of the particle size that is most insensitive to experimental noise. Before loading the samples into a low volume quartz cuvette (QS, 3 $\mathrm{mm}$ ), they were filtered using a syringe filter (Corning Incorporated, Germany) with a pore size of $0.2 \mu \mathrm{m}$, and continuous measurements were started at a fixed temperature. After $90 \mathrm{~min}$, an amount of $\mathrm{Na}_{2} \mathrm{CO}_{3}$ was added, appropriate for a Da5 sample (corresponding to full deacetylation), and continuous measurement of the particle size was continued to monitor the particle aggregation induced by the deacetylation reaction. This procedure was repeated for a number of temperatures and initial KGM concentrations.

\subsection{Atomic force microscopy (AFM)}

Dilute solutions of native $\mathrm{KGM}(0.001 \%, \mathrm{w} / \mathrm{v})$ and the corresponding dilute samples to which an amount of $\mathrm{Na}_{2} \mathrm{CO}_{3}$ was added appropriate for modification corresponding to a Da5 sample, were incubated at, respectively, $25^{\circ} \mathrm{C}, 50{ }^{\circ} \mathrm{C}$ and $75^{\circ} \mathrm{C}$, for $2 \mathrm{~h}$.

Droplets of $5 \mu \mathrm{L}$ of the incubated samples of native and deacetylated KGM were deposited onto freshly cleaved mica surfaces. After incubation for $5 \mathrm{~min}$, weakly attached particles were removed by gentle rinsing with $1 \mathrm{~mL}$ of $0.2 \mu \mathrm{m}$ filtered Milli-Q water. Finally, the samples were slowly dried using $\mathrm{N}_{2}$. Dried KGM samples on mica were imaged with a Digital Instruments NanoScope V at room temperature, using the ScanAsyst ${ }^{\mathrm{TM}}$ mode in air and using silicon nitride cantilevers. Images were collected at 512 lines per image at scan rate of $0.5 \mathrm{~Hz}$. Processing of images consisted only of flattening (NanoScope Analysis 1.5 software).

\section{Results and discussion}

\subsection{Linear viscoelasticity during KGM gelation process}

As is well known, following heating and deacetylation, KGM forms elastic gels, with a modulus that increases during heating (Tye, 1991). This is illustrated in Fig. 1, which shows the development of the storage and loss moduli $G^{\prime}(\omega)$ and $G^{\prime \prime}(\omega)$ at an oscillation frequency of $1 \mathrm{~Hz}$, as a function of the temperature $T$, during a heating ramp with constant heating rate of $1{ }^{\circ} \mathrm{C} / \mathrm{min}$. Immediately before the start of the heating ramp, different amounts of alkali had been added, corresponding to different final degrees of deacetylation (DD). For the case of KGM with lower DD (Da0, Da1 and Da2), the loss factor $\tan \delta$ increases with increasing temperature up to $90^{\circ} \mathrm{C}$, indicating that no gel was formed in the heating process for these (final) degrees of deacetylation. In contrast, for KGM with higher DD (samples Da3, Da4 and Da5), the loss factor tan $\delta$ initially increases with increasing temperature, but then quite rapidly starts decreasing. We deduce approximate gelation temperatures $T_{\text {gel }}$ for these samples as the temperatures for which $\tan \delta=1$. Gelation temperatures for the samples Da3, Da4 and Da5 were, respectively, $T_{\text {gel }}=$ $89^{\circ} \mathrm{C}, 78^{\circ} \mathrm{C}$ and $68^{\circ} \mathrm{C}$, such that gelation temperatures decrease with increasing DD, as expected (Du et al., 2012).

Next we study in more detail the gelation of the Da5 (almost fully deacetylated) sample. Both the storage and loss moduli $G^{\prime}(\omega)$ and $G^{\prime \prime}(\omega)$ were measured $(\omega=1 \mathrm{~Hz})$ after the addition of $\mathrm{Na}_{2} \mathrm{CO}_{3}$ and rapid

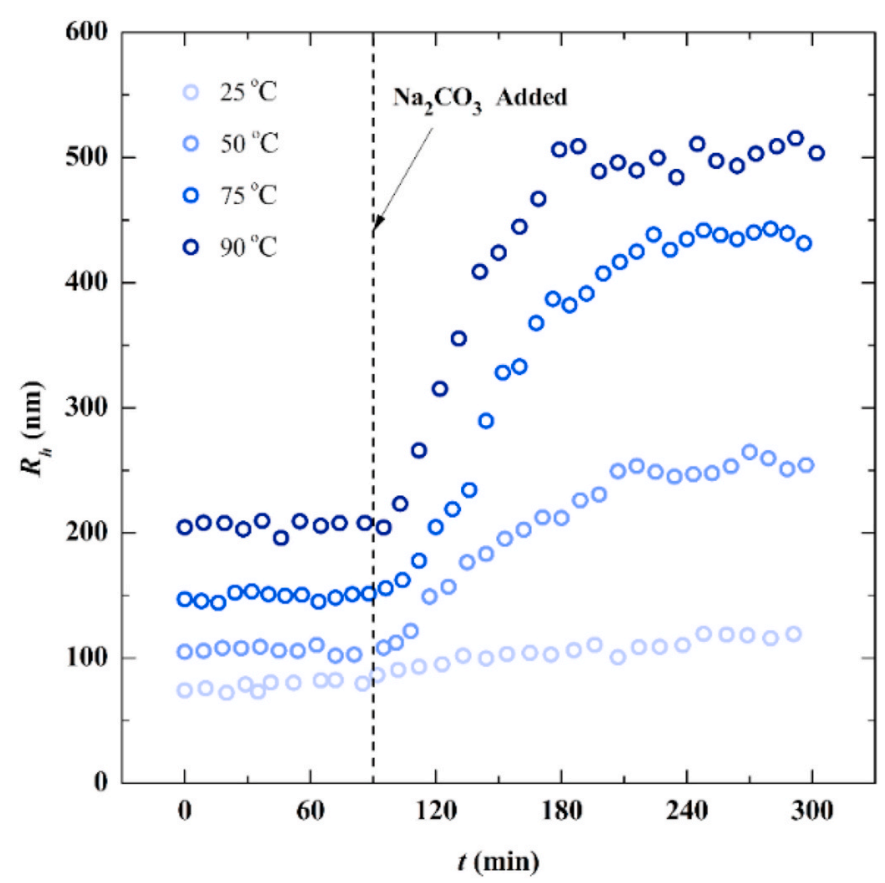

Fig. 3. Z-averaged hydrodynamics radius $R_{h}$ of native KGM $(0.01 \%$ in water) particles as a function of time $t$ during deacetylation reaction at different temperatures. The samples $(0.01 \%$ in water) were firstly measured at different temperatures for $90 \mathrm{~min}$ to get equilibrium and obtain the effects of temperature on KGM particle size, and then $\mathrm{Na}_{2} \mathrm{CO}_{3}$ was added at $t=90 \mathrm{~min}$ and this started the deacetylation reaction at various temperatures.

heating to a fixed temperatures $T_{f}$ and then maintaining the temperature $T_{f}$ until the gelation process has completed. Representative results for the development of the storage and loss moduli as a function of time for selected fixed temperatures are shown in Fig. 2(A)-(C). Final storage and loss moduli $G^{\prime}(\omega)$ and $G^{\prime \prime}(\omega)$ (at $\omega=1 \mathrm{~Hz}$ ) as a function of the fixed reaction temperature $T_{f}$ are shown in Fig. 2(D). From the latter figure it is very clear that for the $\mathrm{Da} 5$ case there is indeed a critical temperature for the gelation process of $T_{\text {gel }}=70{ }^{\circ} \mathrm{C}$. Du, Li, Chen, \& Li (2012) determined the time dependence of $\mathrm{G}^{\prime}$ for partially deacetylated KGM aqueous solutions at different temperatures ranging from 40 to $60^{\circ} \mathrm{C}$, indicating that the gelation was promoted remarkably by raising temperature.

\subsection{Kinetics of deacetylation-induced KGM aggregation probed using $D L S$}

In order to study the microscopic changes occurring during and after the deacetylation reaction that ultimately lead to gel formation, Dynamic Light Scattering (DLS) was used to measure the hydrodynamic radius $\left(R_{h}\right)$ of KGM particles (which means aggregated molecules) during and after the deacetylation reaction at a fixed temperature $T_{f}$. Low concentrations of KGM were used that will not lead to gelation, but just to aggregation of the KGM particles. For a series of temperature, we first measured $R_{h}$ of the native KGM particles. Then, after $90 \mathrm{~min}$, the appropriate amount of $\mathrm{Na}_{2} \mathrm{CO}_{3}$ was added to achieve the full deacetylation corresponding to $\mathrm{Da} 5$ samples and the measurements were continued. Fig. 3 shows the time evolution of $R_{h}$ at various temperatures.

We find that sizes of initial KGM increase from $\approx 80 \mathrm{~nm}$ at $25{ }^{\circ} \mathrm{C}$ to $\approx 200 \mathrm{~nm}$ at $90^{\circ} \mathrm{C}$ (during first $90 \mathrm{~min}$ ). This swelling behavior suggests that polymer-polymer interactions decrease with increasing temperature and polymer-solvent interactions become more favorable (Kruk, Kaczmarczyk, Ptaszek, Goik, \& Ptaszek, 2017). Coil sizes we find in our aqueous solvents (especially at high temperature) are significantly larger than those found from SEC-MALLS in DMSO with 0.5\%w/w $\mathrm{LiBr}$ 

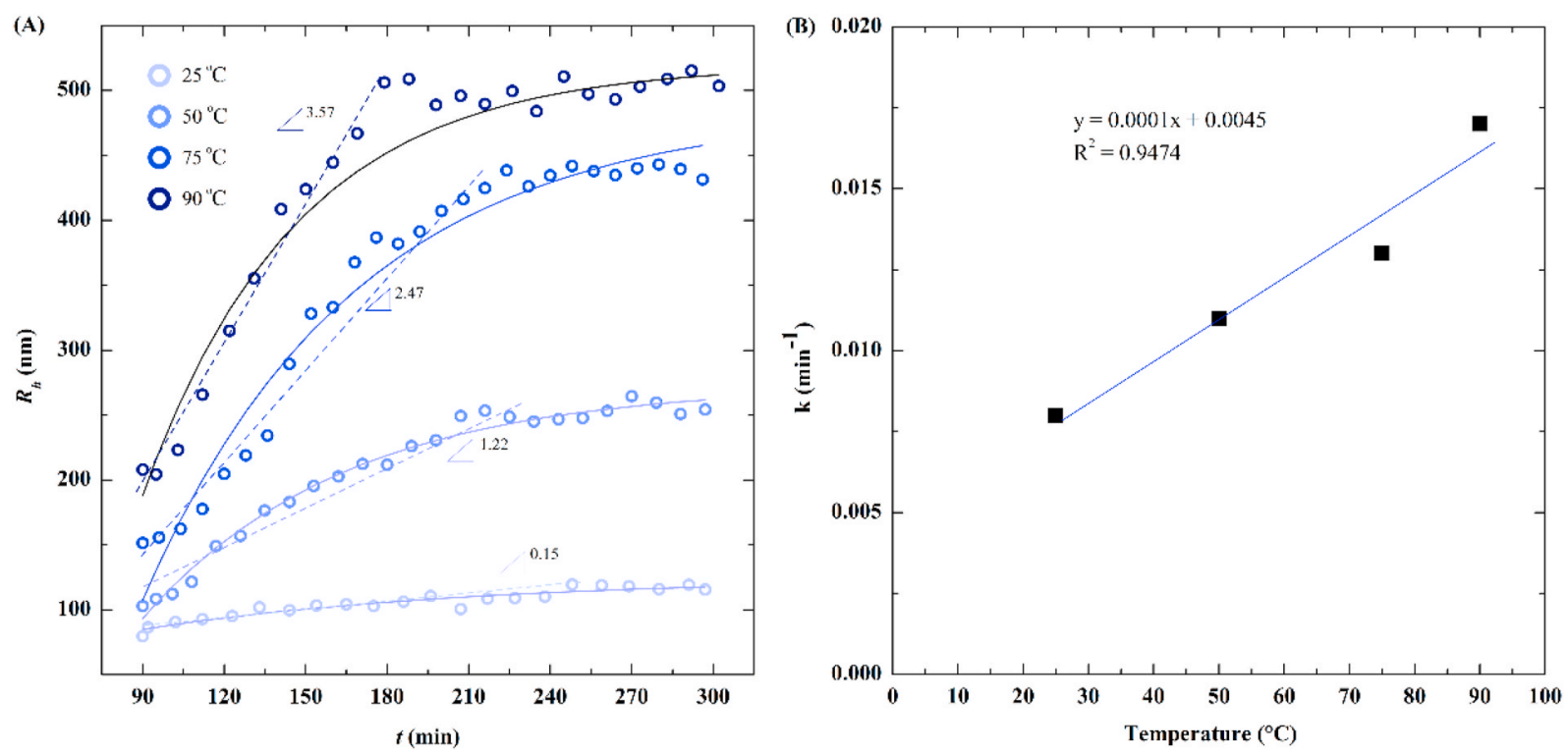

Fig. 4. Aggregation of native KGM $(0.01 \%$ in water $)$ in the presence of $\mathrm{Na}_{2} \mathrm{CO}_{3}$ at different temperature levels. Fitting curves were obtained by Origin Software 8.6 .

(SI Table S1). Hence, it is very likely that the KGM in water seems to form clusters of presumably a small number of KGM molecules.

After addition of the alkali, the KGM particles start to aggregate with rates that strongly increase with increasing temperature. At the lowest temperature of $25^{\circ} \mathrm{C}$, aggregation is still detectable, but it is very slow, and the extent of aggregation is very limited. In contrast, at the highest temperature, aggregation is rapid and the particles become as large as $500 \mathrm{~nm}$. Note that in all cases the aggregation levels off at large reaction times.

The light scattering experiment of Fig. 3 very clearly demonstrates that higher temperatures lead to swelling but not aggregation (or gelation at higher concentrations) of non-deacetylated KGM, and that the addition of alkali (or deacetylation) is what leads to particle aggregation (and hence to gelation at higher concentrations).

We found that the increase in the $R_{h}$ as a function of time can be very well described by first order kinetics, i.e., a simple exponential:

$R_{h}(t)=R_{h, 0}+\Delta R_{h}(1-\exp (-k t))$

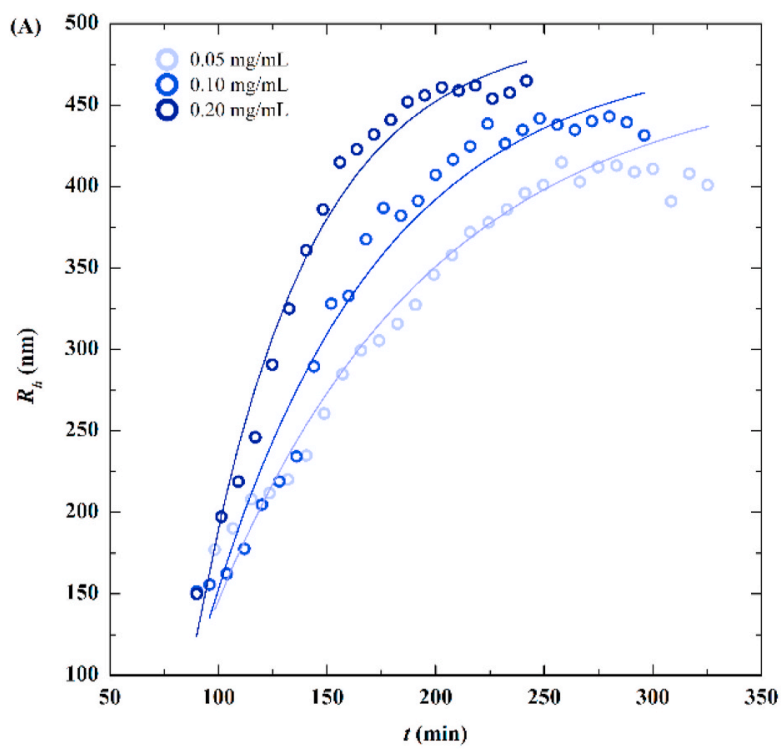

This is shown in Fig. 4A. The rate constant $k$ increases rapidly with increasing temperature $T$, as shown in Fig. 4B. The kinetics of KGM network formation induced by heat and alkali is a two-step reaction: first, glucomannan segments of the KGM are deacetylated, and next, KGM molecules or particles with sufficient deacetylated segments will stick together (Mao et al., 2017):

$\mathrm{MAc} \stackrel{k_{1}}{\rightarrow} \mathrm{M}+\mathrm{Ac}$

$\underbrace{\mathrm{M}+\mathrm{M}+\ldots+\mathrm{M}}_{n} \stackrel{k_{2}}{\rightarrow} M_{n}$

where MAc stands for KGM with acetyl group (Ac), while M denotes the deacetylated KGM.

The first order reaction kinetics that we observe suggest that one of these two steps is in fact rate limiting, thus leading to an overall first order kinetics. Now, if aggregation step would be rate limiting, we would expect a very strong dependence of the rate constants $k$ on

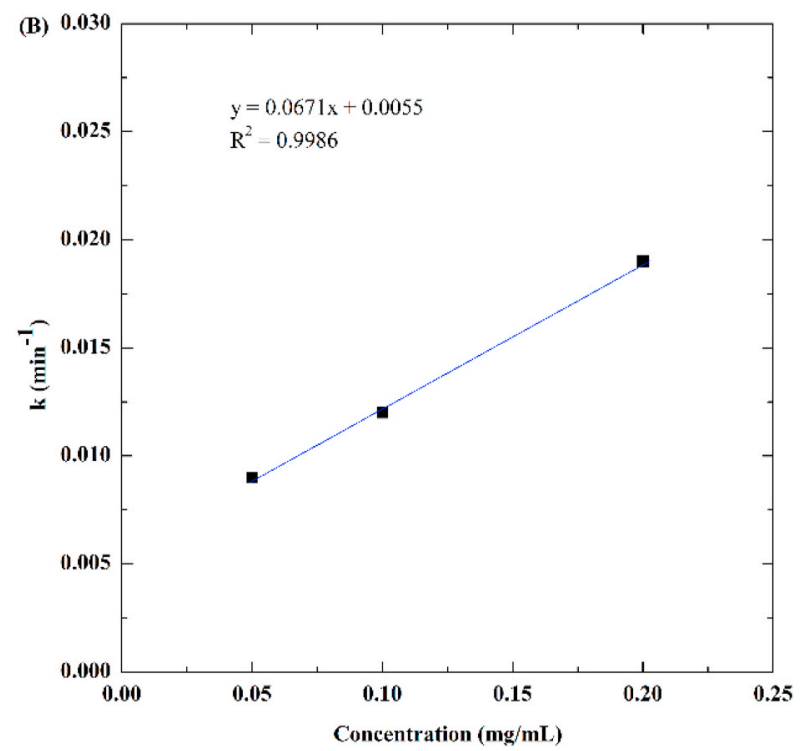

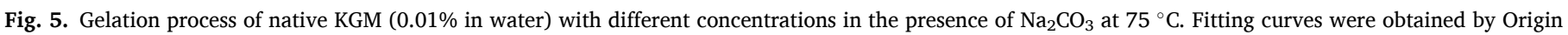
Software 8.6. 

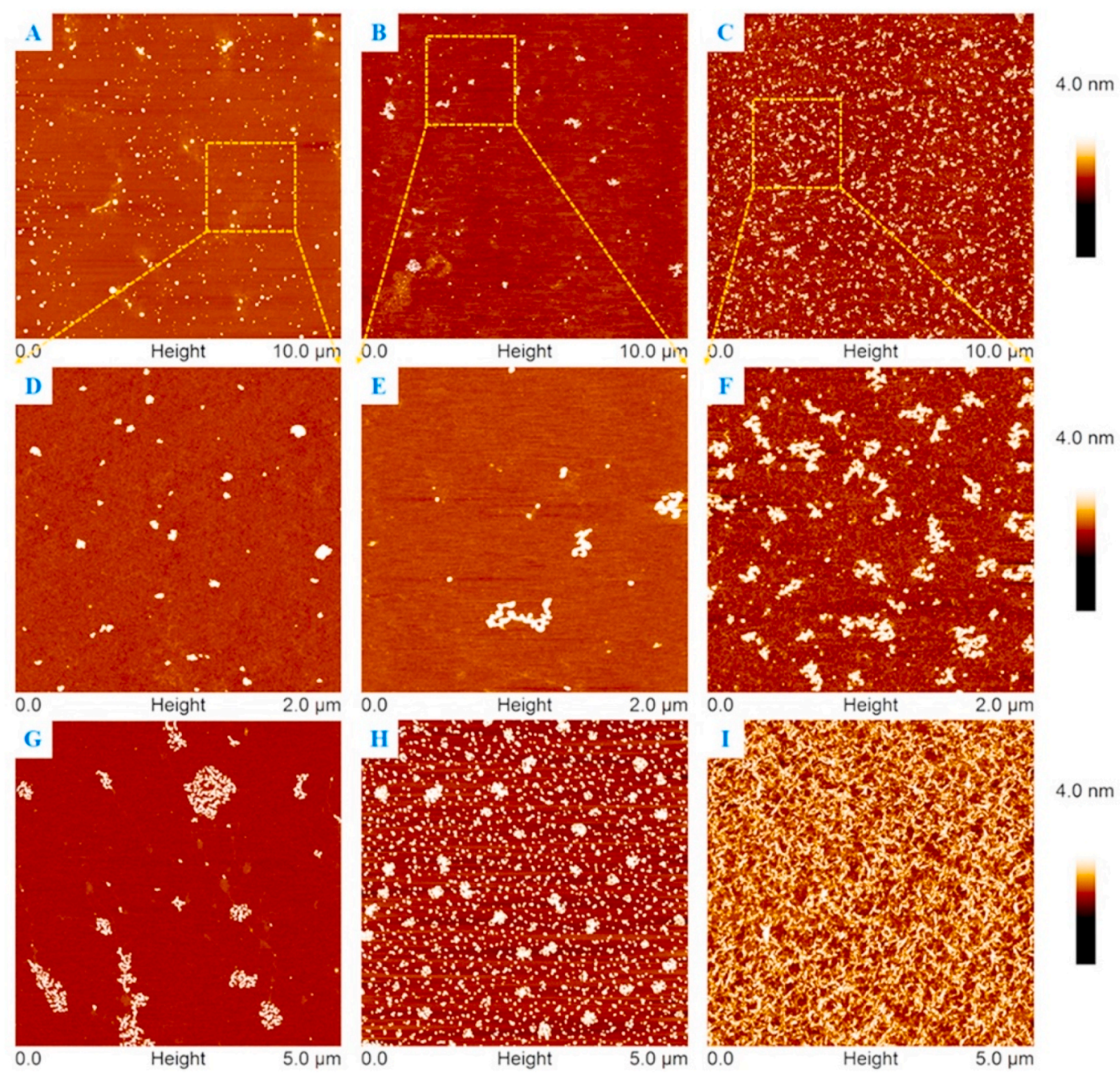

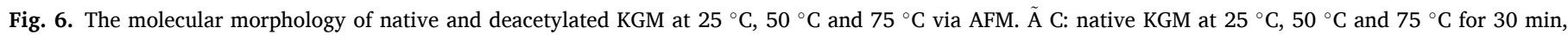
respectively. D F: the zoom area of Ã C, respectively. G I: deacetylated KGM(Da5) at $25{ }^{\circ} \mathrm{C}, 50{ }^{\circ} \mathrm{C}$ and $75{ }^{\circ} \mathrm{C}$ for 30 min, respectively.

concentration. Naively, in fact, we expect that the initial aggregation would be second order in the concentration, since it involves two KGM molecules or particles sticking together. Therefore, we next measured the concentration dependence of the rate constant for the combined of aggregation process (deacetylation step + aggregation step).

Fig. 5A shows the time dependence of the particle size at different concentrations, for a fixed reaction temperature of $75{ }^{\circ} \mathrm{C}$, as well as the fits to simple first order kinetics. The concentration dependence of the rate constant $k$ at a reaction temperature of $75^{\circ} \mathrm{C}$ is shown in Fig. 5B. It is very weak, strongly suggesting that in fact it is the deacetylation reaction that is rate limiting, and that aggregation takes place essentially immediately following deacetylation. The first order kinetics we find here is consistent with the result kinetic modeling of heat and alkali induced KGM aggregation and gelation from Fig. 4A.

\subsection{AFM imaging of KGM aggregation and network formation}

Finally, we used AFM imaging in air of KGM adsorbed on mica to obtain yet more detailed insight in structural changes at the molecular level during heat- and alkali induced aggregation and gelation. While making a connection between the configurations of surface-adsorbed configurations of KGM in dried samples, and its bulk configurations in hydrogels is not straightforward, we nevertheless believe the imaging helps in identifying configurational transitions in KGM upon heating and deacetylation. However, the AFM figures clearly show differences in the molecular morphology of the different samples. First consider the structures observed for native KGM as a function of temperature, Fig. 6A-C and the zooms in Fig. 6D-F. Recall that DLS showed pronounced swelling of native KGM particles with increasing temperature. Consistent with this result, the AFM images show compact objects with sizes of approximate $55.18 \mathrm{~nm}$ for native KGM at room temperature (Fig. 6A, D), and much more extended fibrous objects with sizes of about $123.44 \mathrm{~nm}$ for KGM first heated at $75{ }^{\circ} \mathrm{C}$ and then rapidly adsorbed on the mica (Fig. 6C, F). The latter could be consistent with earlier suggestions that in fact KGM behaves as a semi-flexible polymer (Morris, Adams, \& Harding, 2014).

For KGM samples that had been heated in the presence of alkali, the AFM images of adsorbed KGM look very different (Fig. 6G-I). For KGM incubated in the presence of alkali at $25{ }^{\circ} \mathrm{C}$ (Fig. 6G), we observe extended objects, possibly composed of the much smaller globular KGM particles observed for native KGM at the same temperature, that have aggregated as a consequence of partial deacetylation: since at $25^{\circ} \mathrm{C}$, the deacetylation reaction is very slow, we anticipate that for the AFM sample, it had not yet gone to completion. In contrast, for KGM incubated in the presence of alkali at $75{ }^{\circ} \mathrm{C}$ (Fig. 6I), above the critical gelation temperature for the fully deacetylated $\mathrm{Da} 5$ sample found from rheometry, we observe much thinner fibrous structures that extend over the entire surface. These may correspond to the finite-size fibrous objects also found for native KGM that had swollen at high temperature, but which have now aggregated due to deacetylation, to form a 


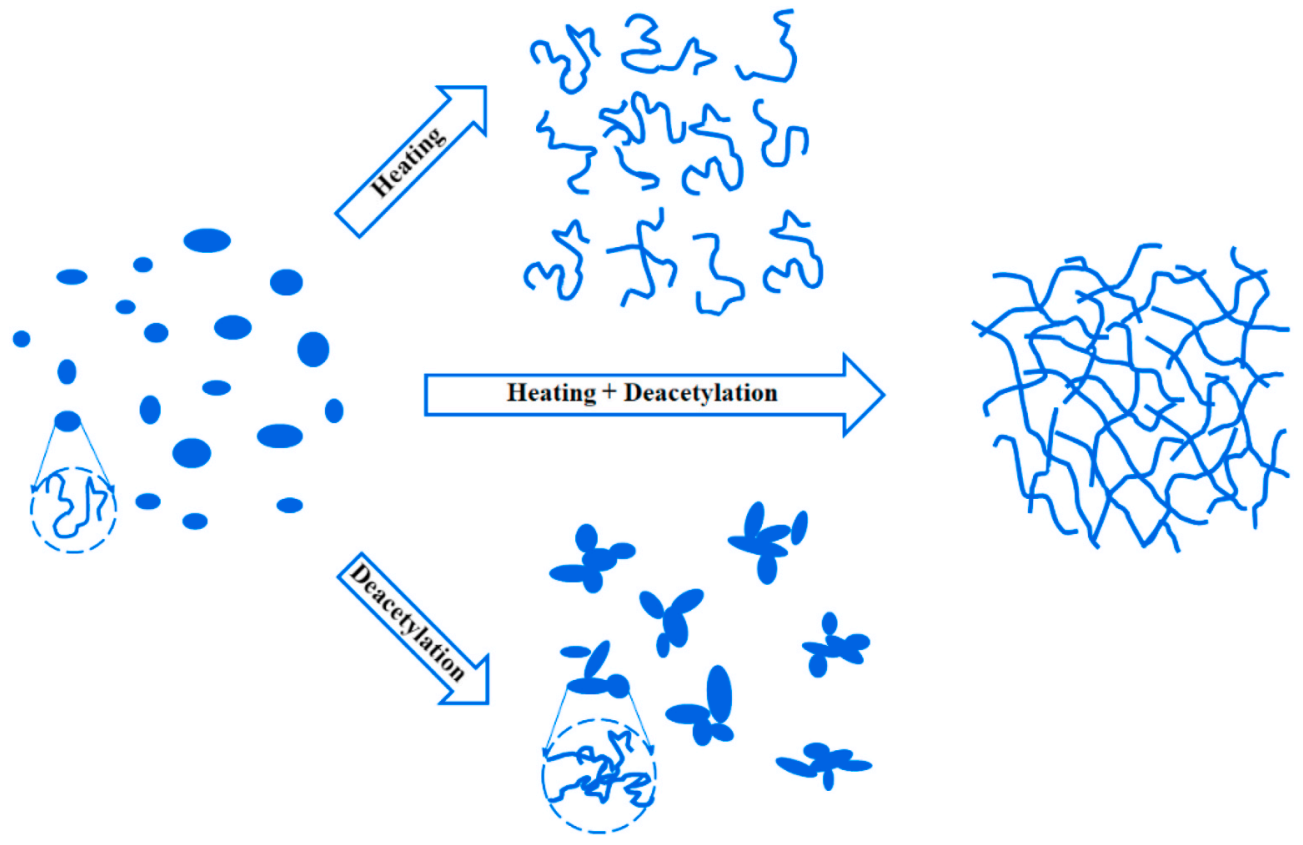

Fig. 7. Schematic illustration of heat and alkali induced gelation process of KGM.

continuous network (Farris et al., 2011).

\section{Conclusions}

AFM has been used extensively to elucidate structural features and changes to them for many other polysaccharides. For example, AFM has been utilized for probing the cation-sensitive aggregation and network formation of the microbial polysaccharide gellan (Ikeda, Nitta, Temsiripong, Pongsawatmanit, \& Nishinari, 2004). AFM imaging has also been helpful in understanding network formation of xanthan gum (Iijima, Shinozaki, Hatakeyama, Takahashi, \& Hatakeyama, 2007). Since AFM by itself can be difficult to interpret, here we have combined it with another microstructural probe, viz., DLS and with macroscale rheology. In agreement with what has been found by other authors, we find that the it is the deacetylation reaction itself which is rate limiting for aggregation and gelation, and that essentially immediately after the reaction, deacetylated groups of regions find each other and form junction zones or crosslinks. The role of temperature in alkali-induced KGM gelation is not only to speed up the deacetylation reaction itself, but we also find that heating leads to significant swelling of KGM, and this swelling is required to eventually form a space filling network. Therefore, gelation only occurs when after the addition of alkali when heating to a sufficiently high temperature. A very simple schematic representing these finding is shown in Fig. 7. Although our work clearly demonstrated the structural changes to KGM during alkali- and heat-induced gelation, we have not addressed in much detail the which molecular forces are responsible for the temperature-dependent swelling and the deacetylation-induced formation of bonds or junction zones among KGM molecules. Also, while the AFM images show fibrous structures that hint a semi-flexible, possibly helical conformations of KGM, confirmation of such configurations would require AFM images at still higher resolutions, combined with other evidence, e.g. spectroscopy.

\section{Author statement}

Tao Zhang: Methodology, Data collection and Original draft preparation. Yong Xue: Conceptualization and Supervision. Renko de Vries: Reviewing and Editing, Supervision. Xiaoqi Xu: Software and Data processing, Supervision. Changhu Xue: Supervision.

\section{Declaration of competing interest}

This manuscript has not been published or presented elsewhere in part or in entirety and is not under consideration by another journal. We have read and understood your journal's policies, and we believe that neither the manuscript nor the study violates any of these. There are no conflicts of interest to declare.

\section{Acknowledgements}

This study is supported by the National Natural Science Foundation of China of China (No. 31571865, 31801496), the Natural Science Fund of Jiangsu Province (No. BK 20180697). Tao Zhang acknowledges the financial support by China Scholarship Council (CSC).

\section{Appendix A. Supplementary data}

Supplementary data to this article can be found online at https://doi. org/10.1016/j.foodhyd.2020.106552.

\section{References}

Case, S., Knopp, J., Hamann, D., \& Schwartz, S. (1992). Characterisation of gelation of konjac mannan using lyotropic salts and rheological measurements. Gums and Stabilizers for the Food Industry, 6, 489-500.

Chen, Li, J., \& Li, B. (2011). Identification of molecular driving forces involved in the gelation of konjac glucomannan: Effect of degree of deacetylation on hydrophobic association. Carbohydrate Polymers, 86(2), 865-871.

Chen, Z.-G., Zong, M.-H., \& Li, G.-J. (2006). Lipase-catalyzed acylation of konjac glucomannan in organic media. Process biochemistry, 41(7), 1514-1520.

Dey, P. M., \& Dixon, R. (1985). Biochemistry of storage carbohydrates in green plants. Academic press.

Du, X., Li, J., Chen, J., \& Li, B. (2012). Effect of degree of deacetylation on physicochemical and gelation properties of konjac glucomannan. Food Research International, 46(1), 270-278.

Farris, S., Schaich, K. M., Liu, L., Cooke, P. H., Piergiovanni, L., \& Yam, K. L. (2011). Gelatin-pectin composite films from polyion-complex hydrogels. Food Hydrocolloids, 25(1), 61-70.

Gao, S., \& Nishinari, K. (2004). Effect of degree of acetylation on gelation of konjac glucomannan. Biomacromolecules, 5(1), 175-185.

Huang, L., Takahashi, R., Kobayashi, S., Kawase, T., \& Nishinari, K. (2002). Gelation behavior of native and acetylated konjac glucomannan. Biomacromolecules, 3(6), 1296-1303. 
Iijima, M., Shinozaki, M., Hatakeyama, T., Takahashi, M., \& Hatakeyama, H. (2007). AFM studies on gelation mechanism of xanthan gum hydrogels. Carbohydrate Polymers, 68(4), 701-707.

Ikeda, S., Nitta, Y., Temsiripong, T., Pongsawatmanit, R., \& Nishinari, K. (2004). Atomic force microscopy studies on cation-induced network formation of gellan. Food Hydrocolloids, 18(5), 727-735.

Jian, W., Siu, K.-C., \& Wu, J.-Y. (2015). Effects of pH and temperature on colloidal properties and molecular characteristics of Konjac glucomannan. Carbohydrate Polymers, 134, 285-292.

Jian, W., Sun, Y., Huang, H., Yang, Y., Peng, S., Xiong, B., et al. (2013). Study on preparation and separation of Konjac oligosaccharides. Carbohydrate Polymers, 92 (2), 1218-1224.

Katsuraya, K., Okuyama, K., Hatanaka, K., Oshima, R., Sato, T., \& Matsuzaki, K. (2003). Constitution of konjac glucomannan: Chemical analysis and 13C NMR spectroscopy. Carbohydrate Polymers, 53(2), 183-189.

Kruk, J., Kaczmarczyk, K., Ptaszek, A., Goik, U., \& Ptaszek, P. (2017). The effect of temperature on the colligative properties of food-grade konjac gum in water solutions. Carbohydrate Polymers, 174, 456-463.

Li, B., \& Xie, B. (2006). Single molecular chain geometry of konjac glucomannan as a high quality dietary fiber in East Asia. Food Research International, 39(2), 127-132.

Maeda, M., Shimahara, H., \& Sugiyama, N. (1980). Detailed examination of the branched structure of konjac glucomannan. Agricultural \& Biological Chemistry, 44(2), $245-252$.
Maekaji, K. (1974). The mechanism of gelation of konjac mannan. Agricultural \& Biological Chemistry, 38(2), 315-321.

Mao, C.-F., \& Chen, C.-H. (2017). A kinetic model of the gelation of konjac glucomannan induced by deacetylation. Carbohydrate Polymers, 165, 368-375.

Morris, G. A., Adams, G. G., \& Harding, S. E. (2014). On hydrodynamic methods for the analysis of the sizes and shapes of polysaccharides in dilute solution: A short review. Food Hydrocolloids, 42, 318-334.

Nishinari, K., Williams, P., \& Phillips, G. (1992). Review of the physico-chemical characteristics and properties of konjac mannan. Food Hydrocolloids, 6(2), 199-222.

Nishinari, K., \& Zhang, H. (2004). Recent advances in the understanding of heat set gelling polysaccharides. Trends in Food Science \& Technology, 15(6), 305-312.

Tye, R. J. (1991). Konjac flour: Properties and applications. Food Technology, 45(3), 82-92.

Williams, M. A., Foster, T. J., Martin, D. R., Norton, I. T., Yoshimura, M., \& Nishinari, K. (2000). A molecular description of the gelation mechanism of konjac mannan. Biomacromolecules, 1(3), 440-450.

Winter, H. H., \& Chambon, F. (1986). Analysis of linear viscoelasticity of a crosslinking polymer at the gel point. Journal of Rheology, 30(2), 367-382.

Xin, C., Chen, J., Liang, H., Wan, J., Li, J., \& Li, B. (2017). Confirmation and measurement of hydrophobic interaction in sol-gel system of konjac glucomannan with different degree of deacetylation. Carbohydrate Polymers, 174, 337-342.

Zhou, Y., Jiang, R., Perkins, W. S., \& Cheng, Y. (2018). Morphology evolution and gelation mechanism of alkali induced konjac glucomannan hydrogel. Food Chemistry, 269, 80-88. 